\title{
Exploration \& Anthropomorphism in Immersive Unit Visualizations
}

\author{
Alexander Ivanov \\ University of Calgary \\ aaivanov@ucalgary.ca

\section{Kurtis Thorvald Danyluk} \\ University of Calgary \\ ktdanylu@ucalgary.ca

\section{Wesley Willett} \\ University of Calgary \\ wesley.willett@ucalgary.ca
}

\begin{abstract}
We report on an initial examination of the potential of immersive unit visualizations in virtual reality, showing how these visualizations can help viewers examine data at multiple scales and support affective, personal experiences with data. We outline unique opportunities for unit visualizations in virtual reality, including support for (1) dynamic scale transitions,

(2) immersive exploration, and (3) anthropomorphic interactions. We then demonstrate a prototype system and discuss the potential for virtual reality visualization to support personal interactions with data.
\end{abstract}

\section{Author Keywords}

Virtual Reality; Data Visualization; Unit Visualization; Scale; Anthropomorphism.

\section{ACM Classification Keywords}

H.5.m. Information interfaces and presentation: Misc.

\section{Introduction}

Permission to make digital or hard copies of part or all of this work for personal or classroom use is granted without fee provided that copies are not made or distributed for profit or commercial advantage and that copies bear this notice and the full citation on the first page. Copyrights for third-party components of this work must be honored. For all other uses, contact the Owner/Author.

CHI'18 Extended Abstracts, April 21-26, 2018, Montreal, QC, Canada (c) 2018 Copyright is held by the owner/author(s).

ACM ISBN 978-1-4503-5621-3/18/04.

https://doi.org/10.1145/3170427.3188544
In 2014, as part of artist Paul Cummins' piece Blood Swept Lands and Seas of Red [6] (Figure 1) 888,246 ceramic poppies surrounded the Tower of London to mark the 100th anniversary of Britain's first day of involvement in the First World War. Each poppy represented a British military fatality resulting from the war. From above, viewers observed a sea of red poppies engulfing the tower, emphasizing the overall 


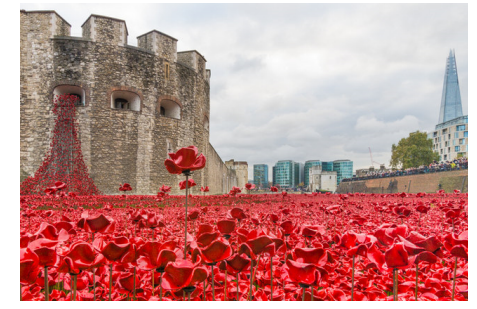

Figure 1: Large art pieces like Blood Swept Lands and Seas of Red [6] use physical objects and people to visualize data in real world environments. Photo (c) Ian Capper (cc-by sa/2.0)

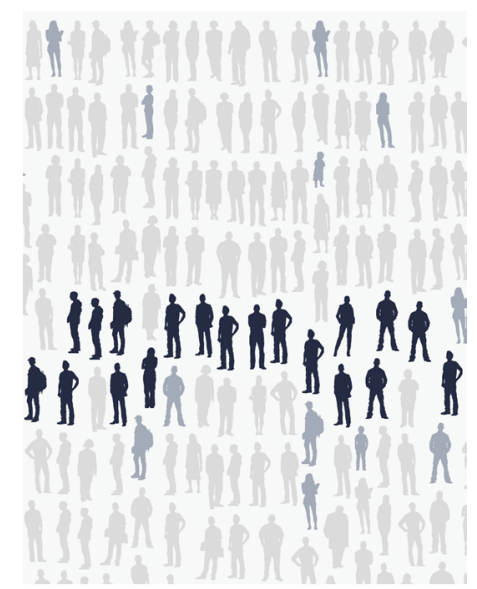

Figure 2: A screenshot of The Washington Post's The Math of Mass Shootings [2] is an example of an anthropomorphic 2D unit visualization. magnitude of British war deaths. However, when walking through the grounds below the tower, viewers could examine each individual poppy in the field, establishing a personal and emotional connection to individual fallen soldiers. Each of these perspectives created a different experience for the viewer as they were immersed in the piece from multiple viewpoints.

Taking inspiration from large visually dramatic art pieces like this and live demonstrations found on the web [7], we examine how virtual reality (VR) tools can be used create immersive unit visualizations that represent data points as objects in virtual space. While this design space is vast, we highlight several unique opportunities that these visualizations provide, including: (1) enabling new experiences with visualizations that span multiple scales, (2) supporting data exploration using physical locomotion and interaction, and (3) prompting affective responses and encouraging storytelling using anthropomorphism.

\section{Related Work}

\section{Unit Visualization}

The practice of using physical objects and visual marks to represent, count, and reason about referents in the world has a rich history that far predates contemporary data visualization. More recently, visualization tools like SandDance [9] have illustrated the potential of dynamic, interactive unit visualizations, which support the examination of much larger datasets by creating visual groupings made up of many individual marks.

\section{VR Information Visualization}

The inherent immersive quality of VR has the potential to create new visualization experiences that were not feasible in the past due to technical constraints of $2 \mathrm{D}$ displays. Recent VR visualization systems such as ImAxes [5] have demonstrated the potential for immersive tools to enable new kinds of spatial exploration and comparison [4].

\section{Immersive Unit Visualization}

Unlike traditional visualizations, which often aggregate multiple values into a smaller number of visual marks, unit visualizations ensure that every data point is represented by a separate visual mark. Extending this concept, we define an "Immersive Unit Visualization" as a three-dimensional unit visualization created for exploration in immersive virtual reality. Bringing unit visualizations into an immersive environment presents a number of unique opportunities, including:

\section{SCALES \& TRANSITIONS}

In physical installations like Blood Swept Lands and Seas of Red viewers must move to another location to change their perspective. By contrast, viewers in an immersive environment can quickly change perspectives and can dynamically adjust the distance between themselves and the visualization. They can also view data from scales and perspectives that are not possible in the physical world, creating entirely new experiences for exploring data. For example, a viewer could choose to experience a visualization at a cityscale or from the perspective of one of the data points.

\section{IMMERSIVE EXPLORATION}

Compared to on-screen displays, immersive virtual reality can greatly increase a viewer's sense of presence (sense of being in an environment) as well as their perceived embodiment within a scene [13]. This allows immersive visualization systems to emulate the exploratory experiences intrinsic to many physical unit 

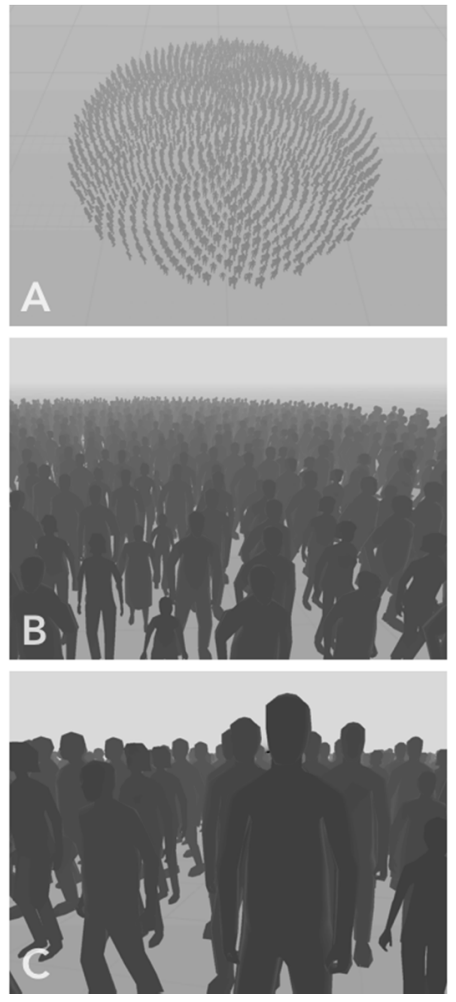

Figure 3: An immersive unit visualization showing mass shooting victims grouped by gender. Viewers can examine the data from different perspectives, observing groupings and patterns from above (top), then zooming in to a human-scale to see details for individual victims (bottom) visualizations and art pieces. We expect this sense of presence can be used to create visualizations that evoke a stronger awareness of scale and space, making it possible to create large virtual environments that reward the same kinds of physical exploration as pieces like Blood Swept Lands and Seas of Red [6]

\section{ANTHROPOMORPHISM}

Research in a variety of fields suggests that the presence or assertion of anthropomorphic characteristics in objects and animals tends to be associated with emotional and empathetic responses [16]. From a visualization perspective, humanoid unit representations are interesting because they are highly recognizable and immediately suggest that the underlying data corresponds to individual people. Moreover, designers can vary the appearance of individual models based on data, altering attributes like height, clothing, gender cues, or in more subtle ways such as via changes in body language.

Recent work by Boy et al. which examined the impact of a variety of different anthropomorphic data graphics (or "anthropographics") in 2D charts found that they of had little to no impact of viewers' level of empathy or willingness to donate to a charitable cause [3]. In contrast to 2D unit visualizations like these, immersive virtual unit visualizations offer the potential for much more with character models that exhibit life-like animations and human-like interactions. This level of fidelity has the potential to enable a kind of direct "face-to-face" relationship with visualizations.

\section{System Design}

We present an initial prototype (Figures 3-5) that allows us to examine the impact of scale transitions, immersive exploration, and anthropomorphism in a visualization of 130 mass shooting incidents in the United States (incidents where four or more people were killed by a shooter) from 1966 to 2017 collected by The Washington Post (Figure 2) [2].

\section{Encoding}

We represent each civilian death in the dataset with a 3D avatar model. To provide more visual differentiation between individuals, we use demographic data to select representative models based on the person's gender and age. Because we lacked character models for very young children, in our visualization we use the same models for infants, children, and teens.

We arrange models in a fractal phyllotaxis pattern [12] in the 3D scene (Figure 4) to ensure even spacing among models. This makes it possible for viewers to move between them while minimizing the need to travel vast distances. We apply a flat, grey shading to the scene to render all figures as silhouettes, creating a ghostly appearance similar to the original 2D piece [2]. Due to a lack of data about each person's identity, this approach also ensured that we did not misrepresent the visual appearance of individual victims.

\section{Interaction}

Viewers can use a two-handed pinch-to-scale gesture to quickly change the scale of the world, allowing them to transition smoothly between a high-level overview and a more detailed, unit-level perspective (Figure 3) When the world is scaled down, viewers can observe general aggregated trends in the data from above

(Figure 4). When the world is scaled up, viewers can walk among the avatars and learn more about them. 


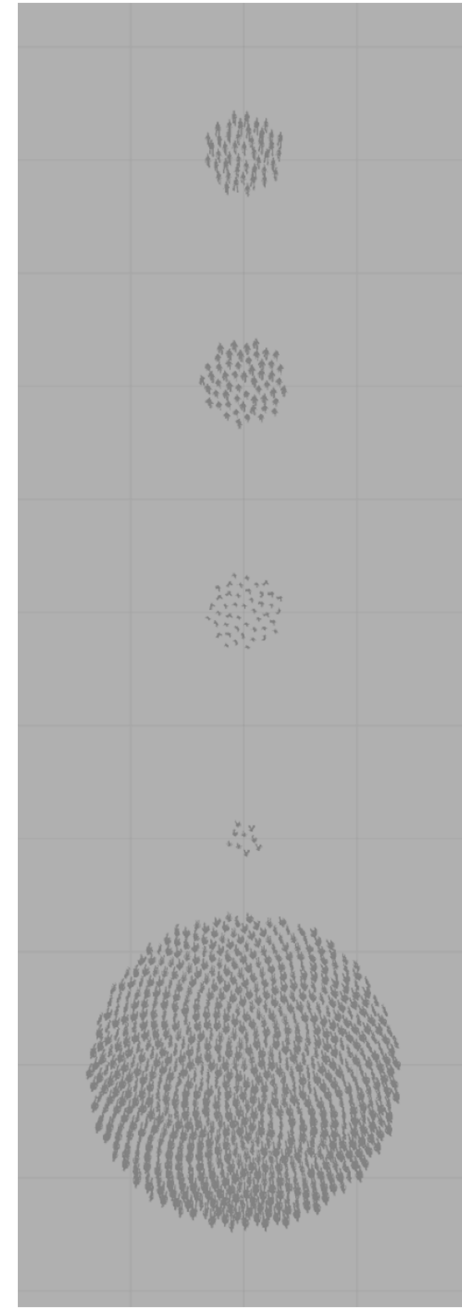

Figure 4: A birds eye view of the victim avatars grouped by age in the visualization.

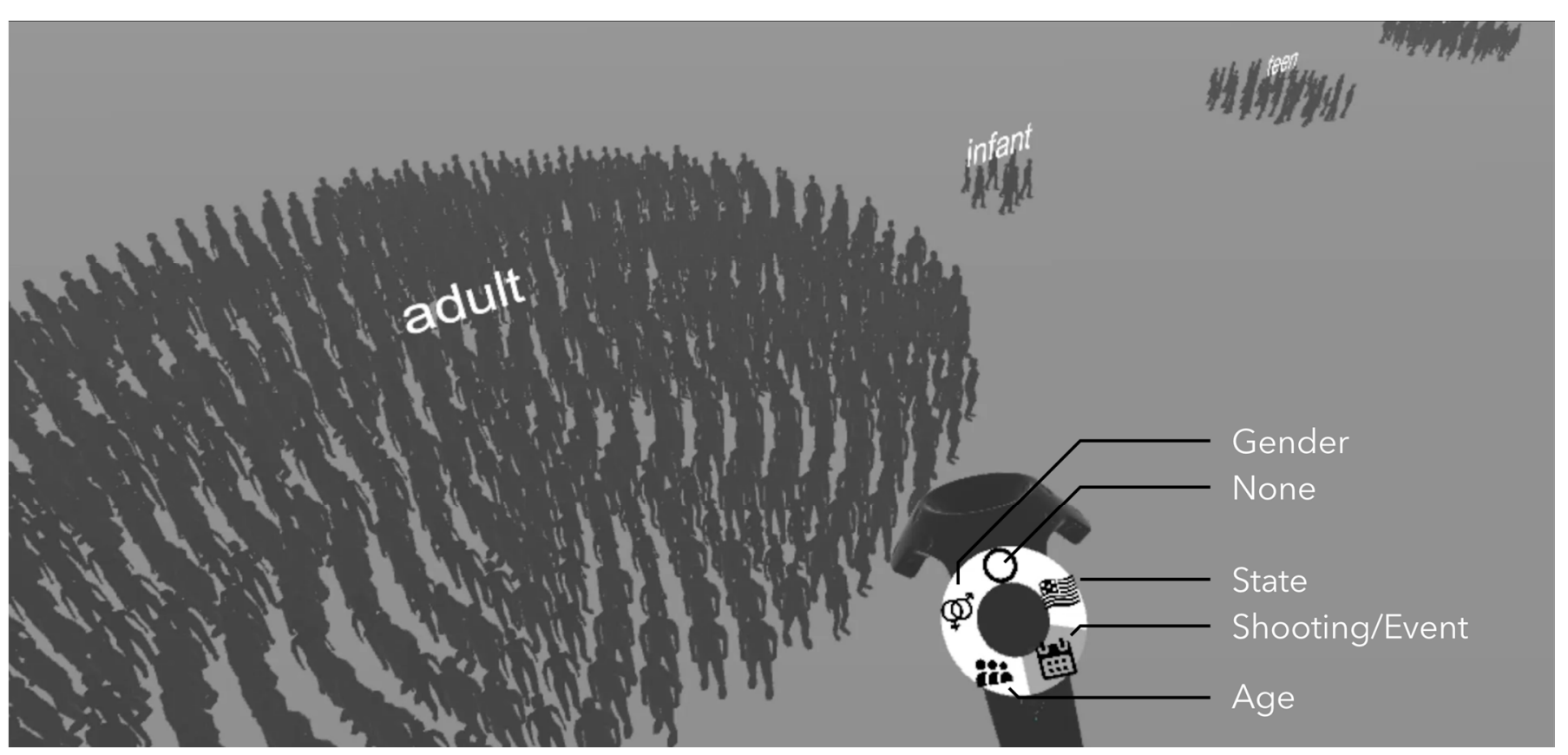

Figure 5. Using the radial menu, viewers can group shooting victims by gender, age group, or shooting event. Viewers can also group the victims by U.S. state or choose to leave them un-grouped. Icons made by Freepik from www.flaticon.com.

Touching one of the avatars and pressing the menu button on either controller triggers a speech bubble describing the shooting victim. Using a radial menu on the right controller trackpad, viewers can also group victims by age, gender, shooting event, or U.S. state, or choose "None" to explore the victims as a single large crowd (Figure 5). Selecting a new grouping option causes the avatars animate and walk together into their new clusters. Large 3D labels above each group of avatars serve as navigational landmarks.

\section{Discussion}

Challenges for VR Unit Visualization

ORIENTATION AND SITUATIONAL AWARENESS

Our initial experiences highlight how the open-world nature of immersive visualizations like the one in our prototype has the potential to become disorienting. Prior work on orientation and wayfinding suggests that landmarks can make it easier for viewers to situate themselves [8]. Immersive visualizations may also benefit from the inclusion of good default viewpoints that allow viewers to reset their perspective by quickly teleporting back to a known, consistent overview. 
VISUAL FIDELITY AND UNIT APPEARANCE

In early versions of the system, we added lowresolution textures on top of the models that made them appear more lifelike. However, this created an unpleasant experience as viewers were confronted by multiple model "clones" which appeared identical, but were intended to represent different individuals. In our particular case, stylizing the scene and rendering the avatars as silhouettes addressed this concern, but also made it more difficult to identify specific victims. Crowd simulation techniques might help address this issue, systematically generating diverse sets of avatars [15].

\section{Personal Space}

Users encountering human-like models in virtual environments often exhibit some of the same nonverbal social cues that exist in the physical world [20]. In our prototype, we observed that changing the grouping of data could cause avatars to walk through the user's body, an act that viewers tended to find unsettling. Furthermore, avatars would often stand uncomfortably close to the user, crossing social boundaries. Learnings from proxemics, including Hall's 'distance zones' [10] may provide useful templates for how personal space should be handled.

Opportunities for VR Unit Visualization

DYNAMIC AND PERSONAL INTERACTIONS

High-quality models have the potential to support more dynamic interactions within avatar-based unit visualizations. In our prototype, viewers can approach individual models and touch them to view their personal stories. Adding more complex agent-based interactions via natural language could add another level of depth to the experience. Characters in the environment could also provide a guided tour of the dataset using storytelling techniques inspired by contemporary first and third-person games. Past research suggests that using empathetic characters in narrative learning tools may indeed lead to heightened perceptions of presence, involvement, and control [11].

\section{WORLDS-IN-MINIATURE}

Immersive visualization environments may also lend themselves well to navigation techniques like world-inminiature [14]. This approach places a miniature copy of the entire environment or visualization in the scene next to the viewer. These worlds-in-miniature could help support overview+detail browsing, allowing viewers to identify higher-level trends and navigate to distant locations in the vis without changing its scale.

NON-ANTHROPOMORPHIC UNITS

Datasets which represent physical objects (museum collections, 3D models, etc.) may also be excellent candidates for immersive unit visualizations, especially where detailed 3D models exist. Here, units in the visualization might serve not just as visual marks that represent the data but instead as direct representations of the data itself. A visitor might filter, sort, group, and explore an entire collection in virtual space-comparing items that might be impossible to physically collocate.

\section{SONIFICATION AND AMBIENT AUDIO}

In addition to creating convincing visual landscapes, unit visualizations could leverage audio mappings to create strong spatial association between visual and audio elements with unique sounds, pitches, or speech that appear to originate directly from individual marks. For example, when a viewer approaches an individual mark, the ambient audio could provide details-ondemand or encode additional quantitative values. 


\section{References}

1. Jeremy N. Bailenson, Jim Blascovich, Andrew C. Beall, and Jack M. Loomis. 2001. Equilibrium

Theory Revisited: Mutual Gaze and Personal Space in Virtual Environments. Presence: Teleoperators and Virtual Environments 10, 6: 583-598.

2. Bonnie Berkowitz, Lazaro Gamio, Denise Lu, Kevin Uhrmacher, and Todd Lindeman. 2017. The Math of Mass Shootings. Retrieved September 10, 2017 from

https://www.washingtonpost.com/graphics/nationa l/mass-shootings-in-america/.

3. Jeremy Boy, Anshul Vikram Pandey, John Emerson, Margaret Satterthwaite, Oded Nov, and Enrico Bertini. 2017. Showing People Behind Data: Does Anthropomorphizing Visualizations Elicit More Empathy for Human Rights Data? Proceedings of the 2017 CHI Conference on Human Factors in Computing Systems - CHI '17, ACM Press, 54625474.

4. Maxime Cordeil, Benjamin Bach, Yongchao Li, Elliott Wilson, and Tim Dwyer. 2017. A Design Space for Spatio-Data Coordination: Tangible Interaction Devices for Immersive Information Visualisation. IEEE Pacific Visualization Symposium (PacificVis 2017): 1-6.

5. Maxime Cordeil, Andrew Cunningham, Tim Dwyer, Bruce $\mathrm{H}$. Thomas, and Kim Marriott. 2017. ImAxes: Immersive Axes as Embodied Affordancesfor Interactive Multivariate Data Visualisation. .

6. Paul Cummins and Tom Piper. 2014. Blood Swept Lands and Seas of Red. Retrieved September 10 2017 from http://dataphys.org/list/tower-poppies888246-ceramic-poppies-to-commemorate-fallensoldiers-in-ww1/.

7. Cut. 2016. Atheists, Christians, Jews, and Muslims on Rights | Dirty Data - Ep 6 | Cut. Retrieved January 12, 2018 from https://www. youtube.com/watch?v=9I0Lba-WUcU.

8. Rudolph P. Darken and Barry Peterson. 2001. Spatial Orientation, Wayfinding, and
Representaion. In Handbook of Virtual Environment Technology. .

9. Steven M Drucker and Roland Fernandez. 2014. A unifying framework for animated and interactive unit visualizations. 1-9.

10. Edward T. Hall. 1966. The Hidden Dimension. Anchor.

11. Scott W. McQuiggan, Jonathan P. Rowe, and James C. Lester. 2008. The effects of empathetic virtual characters on presence in narrative-centered learning environments. Proceeding of the twentysixth annual $\mathrm{CHI}$ conference on Human factors in computing systems - CHI '08, ACM Press, 1511.

12. Petra Neumann, S. Carpendale, and A. Agarawala. 2004. PhylloTrees: Phyllotactic Patterns for Tree Layout. IEEE Symposium on Information Visualization, IEEE, p3-p3.

13. M. Slater and M. Usoh. Presence in immersive virtual environments. Proceedings of IEEE Virtual Reality Annual International Symposium, IEEE, 9096.

14. Richard Stoakley, Matthew J. Conway, and Randy Pausch. 1995. Virtual reality on a WIM.

Proceedings of the SIGCHI conference on Human factors in computing systems - CHI'95, ACM Press, 265-272.

15. Daniel Thalmann, Helena Grillon, Jonathan Maim and Barbara Yersin. 2009. Challenges in Crowd Simulation. 2009 International Conference on CyberWorlds, CW '09, 1-12.

16. Jeroen Vaes, Federica Meconi, Paola Sessa, and Mateusz Olechowski. 2016. Minimal humanity cues induce neural empathic reactions towards nonhuman entities. Neuropsychologia 89: 132-140.

17. Laurie M. Wilcox, Robert S. Allison, Samuel Elfassy, and Cynthia Grelik. 2006. Personal space in virtual reality. ACM Transactions on Applied Perception 3, 4: $412-428$ 